\title{
Ozonated autohemotherapy modulates the serum levels of inflammatory cytokines in gouty patients
}

This article was published in the following Dove Press journal:

Open Access Rheumatology: Research and Reviews

II August 2017

Number of times this article has been viewed

\author{
Lian-Yun $\mathrm{Li}^{1,2}$ \\ Ruo-Lan $\mathrm{Ma}^{3}$ \\ Liqin $\mathrm{Du}^{4}$ \\ An-Shi Wu ${ }^{5}$ \\ 'Department of Anesthesiology, \\ Dongfang Hospital of Beijing \\ University of Chinese Medicine, \\ ${ }^{2}$ Department of Pain, Beijing Electric \\ Power Hospital, ${ }^{3}$ Department of \\ Anesthesiology, Beijing Stomatological \\ Hospital, Capital Medical University, \\ Beijing, People's Republic of China; \\ ${ }^{4}$ Department of Chemistry \& \\ Biochemistry, Texas State University, \\ San Marcos, TX, USA; ${ }^{5}$ Department \\ of Anesthesiology, Beijing Chao-Yang \\ Hospital, Capital Medical University, \\ Beijing, People's Republic of China
}

Correspondence: Liqin Du Department of Chemistry \& Biochemistry, Texas State University, 60I University Drive, San Marcos,

TX 78666, USA

$\mathrm{Tel}+|\mathbf{5}| 22451038$

Fax + I 5122452374

Email liqindu6@txstate.edu

An-Shi Wu

Department of Anesthesiology, Beijing

Chao-Yang Hospital, Capital Medical

University, 8 Gongti South Road,

Chaoyang District, Beijing 100020,

People's Republic of China

Tel $+86108523 \quad 1200$

Fax +86 I0 $8523 \quad 1212$

Email wuanshicmu@I63.com
Objectives: Ozonated autohemotherapy (O3-AHT) has been used to effectively treat gout, but the underlying therapeutic mechanisms remain unknown. In this study, as an initial effort to understand the therapeutic mechanisms of O3-AHT, we aim to examine the effect of O3-AHT on serum inflammatory cytokine levels in gouty patients.

Patients and methods: Three groups of patients and healthy subjects were recruited, including the gouty $(n=10)$, hyperuricemia $(n=10)$, and healthy control $(n=11)$ groups. Cytometric bead array was applied to examine 12 cytokines before (T0), during (T1), and after (T2) therapies. Results: Three cytokines, IL-8, IL-12, and MCP-1, were detectable in all participants. Before O3-AHT, the average serum levels of IL-8 and MCP-1 were higher in the gout group than in the hyperuricemia and healthy control groups, confirming the inflammation status in gouty patients. After the 5th course of O3-AHT (T1), IL-8 level was significantly increased compared to that at T0. IL-12 level was also raised at T1, although the difference did not reach statistical significance. After completing the therapy, both IL-8 and IL-12 levels decreased to levels lower than those at T0. MCP-1 level remained essentially unchanged during and after treatment.

Conclusion: Our results indicate that $\mathrm{O} 3$-AHT induces a significant change in serum cytokine levels, suggesting that modulating the inflammatory process is one of the therapeutic mechanisms underlying O3-AHT. In addition, the sensitive response of serum IL-8 and IL-12 levels to O3-AHT suggests that these cytokines may be developed as biomarkers to evaluate the therapeutic effect of O3-AHT in gouty patients.

Keywords: gout, ozonated autohemotherapy, inflammation, cytokine

\section{Introduction}

Gout is a common inflammatory arthritis induced by an increased serum urate level and is characterized by chronic depositions of monosodium urate (MSU) crystals in tissues and synovial fluid. ${ }^{1}$ Hyperuricemia, acute arthritis, and tophus are typical clinical manifestations of gout. ${ }^{2}$ Risk factors for gout have been identified. For example, high intake of purine-rich food, beverages, alcohol, and fructose and intake of certain medications such as aspirin have been demonstrated to increase the risk of gout. ${ }^{3,4}$ Metabolic syndromes, such as hypertension, diabetes, and obesity, are also acknowledged risk factors for hyperuricemia and gout. ${ }^{4}$ Recent genome-wide association studies have suggested that genetic variations in SLC2A9/GLUT9, ABCG2, and URAT1/SLC22A12, three genes that play roles in urate transportation, are associated with an increased risk of gout. ${ }^{5}$

Despite the clear identification of the risk factors, the prevention and therapy of gout remain to be challenging and the incidence rate of gout remains high. Epidemiological studies revealed that the incidence and prevalence of gout have kept rising for at least 
four decades. In the United States, the prevalence of gout is $4 \%$, affecting $\sim 8.3$ million adults, which has increased two- to fourfold or more since 1970s. Meanwhile, the prevalence of gout in the UK is $1.4 \%{ }^{6}$

Anti-inflammation and urate-lowering therapies are the main therapeutic principles. ${ }^{7}$ Colchicine, nonsteroidal antiinflammatory drugs, glucocorticoid, allopurinol, probenecid, and febuxostat have been applied in long-term therapy. ${ }^{7}$ However, coexistence of underlying diseases with gout and contraindication leads to inefficiency of conventional pharmacotherapy.

The efficacy of ozonated autohemotherapy (O3-AHT) has been indicated in several types of disease, including hepatitis, ${ }^{8}$ ischemic disease, and retinal degenerative diseases. ${ }^{9}$ The therapeutic potential of O3-AHT for gout was implicated but has not been intensively investigated. In our previous study, the safety and therapeutic efficacy of O3-AHT for gouty patients were investigated. ${ }^{10}$ We found that the creatinine clearance ratios were significantly increased and the pain visual analog scale scores were significantly decreased after O3-AHT. ${ }^{10}$ These results indicate that O3-AHT could improve renal function and relieve pain in gouty patients.

Gout is an MSU-induced aseptic inflammation characterized by disorder of inflammatory cytokines. ${ }^{1,11}$ NLRP3 inflammasome activated by MSU recruits a second cascade of the inflammatory process, and IL-1 released from leukocytes triggers a cascade of biochemical events that involve multiple crucial cytokines and mediators. ${ }^{12}$ Blocking this multistep inflammatory process involves various cellular and biochemical events, such as crystal coating, apoptotic cell clearance, orchestration of proinflammatory cytokine, and release of TGF- $\beta 1,{ }^{13,14}$ and is one of the mechanisms of gout therapy. Although our previous study has suggested the efficacy of O3-AHT in reducing pain and in improving renal function, no study has been conducted to investigate the effect of O3-AHT on the inflammatory status in gouty patients. Furthermore, little is known concerning the mechanisms and pathways by which O3-AHT reduces gouty symptoms. Our hypothesis is that O3-AHT of gout patients reduces the inflammation response, leading to the change in the serum cytokine expression levels. The objective of this study is to determine the effect of O3-AHT on the serum levels of inflammatory cytokines in gouty patients.

\section{Patients and methods}

\section{Patients' characteristics}

Three groups of patients and healthy subjects were recruited, including the gouty $(n=10)$, hyperuricemia $(n=10)$ and healthy control $(n=11)$ groups. The screening criteria for gouty patients were established according to the European League Against Rheumatism (EULAR) diagnosis standard of gout. ${ }^{15}$ Exclusion criteria included all main nongouty conditions known to change urate level, as we described in our published work. ${ }^{10}$ The demographic characteristics of the 10 gouty patients were reported in the previous study. ${ }^{10}$ Eleven healthy participants were recruited as healthy controls. All clinical data were collected from medical records and routine examinations.

\section{Patient treatment}

All the gouty patients received O3-AHT for 1-4 weeks and were subsequently followed up for 5-28 weeks. Ozone generator (Hyper Medonzon comfort; HERRMANN Apparatebau GmbH, Elsenfeld, Germany) and Solar 8000M monitor (GE Healthcare, Pittsburgh, PA, USA) were applied for O3-AHT. A total of $200 \mathrm{~mL}$ of blood from participants was mixed with $20 \mathrm{~mL}$ of $3.8 \%$ sodium citrate and exposed to an oxygenozone mixture at a concentration of $50 \mu \mathrm{g} / \mathrm{mL}$ for 5 minutes. Subsequently, all peripheral blood was transfused back to the same participant. O3-AHT was performed three times a week, for 10 times in total. The healthy individuals in the control group did not receive any medical treatment.

\section{Cytokine analysis assay}

The serum levels of IFN- $\gamma$, TGF- $\beta$, IL- $1 \alpha$, IL-1 $\beta$, IL-2, IL-4, IL-6, IL-10, IL-13, MCP-1, IL-8, and IL-12 in patients were measured using the cytometric bead array following the manufacturer's protocol. Measurements were conducted before therapy (T0), during therapy (after 5th course of O3-AHT, 1-4 weeks, T1), and after therapy (after 10th course of O3-AHT, 5-28 weeks, T2).

\section{Statistics}

SPSS software, Version 17.0 (SPSS, Chicago, IL, USA) was applied for analysis. All the data were represented as mean \pm SD. Paired Wilcoxon signed-rank test was used when comparing data obtained at T0, T1, and T2, which were all with nonnormal distribution or unequal variance. Chi-square test was used to compare the three groups with nonnormal distribution. For all analyses, $P<0.05$ was considered as statistically significant.

\section{Ethics and consent}

All participants provided written informed consents, and the study was approved by the Ethics and Academic Committees of Capital Medical University. All gouty patients received standard O3-AHT at the Department of Pain Therapeutic Center, Xuanwu Hospital, Capital Medical University. 


\section{Results}

\section{General characteristics of patient treatment}

Of the 10 gouty patients, nine patients finished 10 courses of O3-AHT and one patient finished seven courses of treatment. No patients had routinely taken drugs that might affect uric acid for at least 6 weeks before and during this research period. All the 10 gouty patients completed the protocol for serum cytokine measurement before (T0), during (T1), and after (T2) O3-AHT. The participants in the hyperuricemia group $(n=10)$ and healthy control group $(n=11)$ received one-time serum cytokine measurements for the comparison with gouty patients before O3-AHT.

\section{The average serum levels of IL- 8 and MCP-I in gouty patients prior to O3-AHT are higher than those in hyperuricemia patients and healthy control individuals}

In order to determine the expression levels of inflammatory cytokines in gouty patients without O3-AHT, we measured

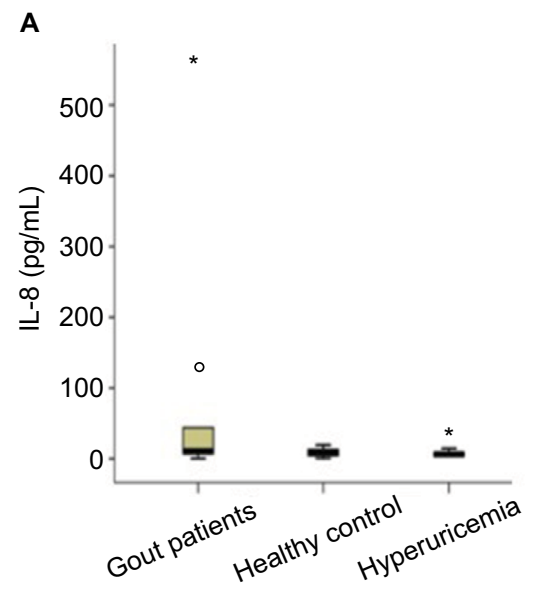

the serum levels of 12 cytokines in gouty patients before they were treated with O3-AHT (T0) and compared these with the hyperuricemia and healthy control individuals. As shown in Figure 1 and Table 1, the average serum levels of IL-8 and MCP-1 are higher in gouty patients than in hyperuricemia patients and healthy control individuals, although the differences did not reach statistical significance. The average serum level of IL-12 is lower in gouty patients than in hyperuricemia and healthy controls, and the differences did not reach statistical significance either.

Table I Serum cytokine levels in gouty patients, hyperuricemia patients, and healthy control individuals before O3-AHT

\begin{tabular}{|c|c|c|c|c|}
\hline \multirow[t]{2}{*}{ Cytokine } & \multicolumn{3}{|c|}{ Cytokine levels (mean \pm SD) } & \multirow{2}{*}{$\begin{array}{l}\text { Chi- } \\
\text { square } \\
\text { test }\end{array}$} \\
\hline & Gout & $\begin{array}{l}\text { Healthy } \\
\text { control }\end{array}$ & Hyperuricemia & \\
\hline $\begin{array}{l}\mathrm{IL}-8 \\
(\mathrm{pg} / \mathrm{mL})\end{array}$ & $77.95 \pm 171.95$ & $9.31 \pm 9.21$ & $9.04 \pm 6.36$ & $\begin{array}{l}\chi^{2}=2.017, \\
P=0.365\end{array}$ \\
\hline $\begin{array}{l}\text { MCP-I } \\
(\mathrm{pg} / \mathrm{mL})\end{array}$ & $95.89 \pm 52.00$ & $72.23 \pm 43.81$ & $78.53 \pm 65.99$ & $\begin{array}{l}\chi^{2}=1.503, \\
P=0.472\end{array}$ \\
\hline $\begin{array}{l}\mathrm{IL}-12 \\
(\mathrm{pg} / \mathrm{mL})\end{array}$ & $1.66 \pm 2.7 \mathrm{I}$ & $3.07 \pm 2.98$ & $2.52 \pm 3.48$ & $\begin{array}{l}\chi^{2}=2.834, \\
P=0.242\end{array}$ \\
\hline
\end{tabular}

Abbreviations: O3-AHT, ozonated autohemotherapy; SD, standard deviation.

B

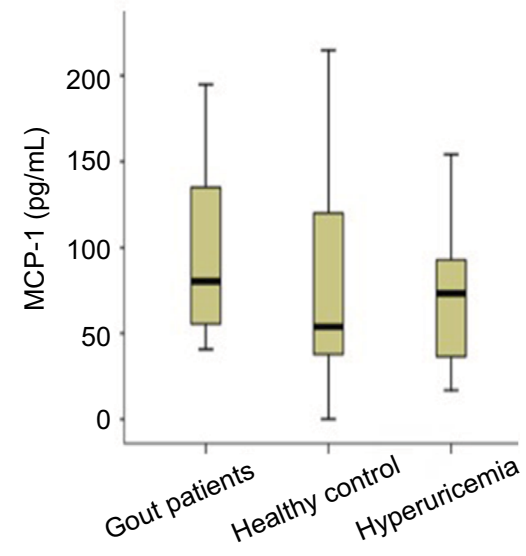

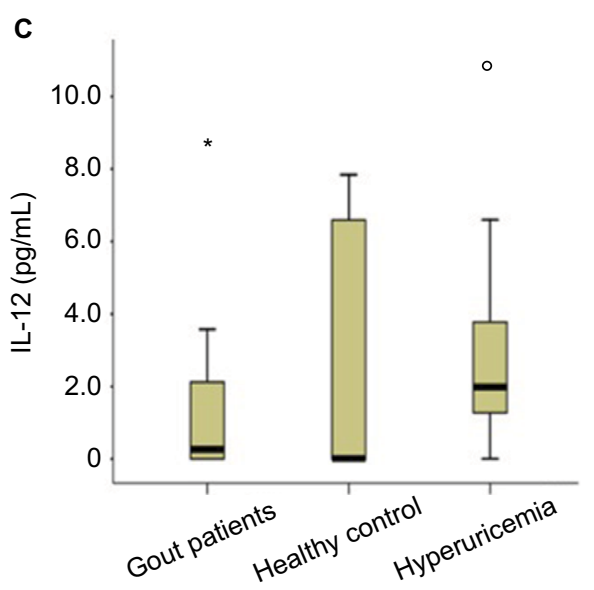

Figure I Serum cytokine levels in gouty patients, healthy controls, and hyperuricemia patients.

Note: Shown are the mean \pm SD of the serum IL-8 (A), MCP-I (B), and IL-I2 (C) levels in the indicated groups. ${ }^{\circ}$ and * are the samples that have dramatic higher cytokine levels than the rest of the samples in the corresponding group; these samples have the most significant impact on the mean and SD in the corresponding group. 
The rest of the nine cytokines that we examined, including IFN- $\gamma$, TGF- $\beta$, IL- $1 \alpha$, IL-1 $\beta$, IL-2, IL-4, IL-6, IL-10, and IL-13, were undetectable in all the three groups.

\section{The serum IL-8 level in gouty patients} was significantly increased after the 5 th course of O3-AHT (TI) and then decreased after completing O3-AHT (T2) In order to examine whether O3-AHT modulates the inflammation status in gouty patients, we measured the serum levels of all the 12 cytokines following O3-AHT. Consistent with the above observations with patients without treatment, only three cytokines, IL-8, MCP-1, and IL-12, were detectable. The rest of the nine cytokines were still undetectable after O3-AHT.

We first analyzed the effect of O3-AHT on serum IL-8 level. As shown in Figure 2 and Table 2, after the 5th course of O3-AHT (T1), the IL-8 level significantly increased to $262.52 \mathrm{pg} / \mathrm{mL}$ compared to the level of $77.95 \mathrm{pg} / \mathrm{mL}$ prior to O3-AHT ( $Z=2.487, P=0.013$ ). Follow-up measurement showed that IL-8 level dramatically decreased to $45.83 \mathrm{pg} /$ $\mathrm{mL}$ after completing O3-AHT (T2); this level was lower than the IL-8 level prior to O3-AHT, and the difference reached marginal statistical significance $(Z=1.376, P=0.169$, comparing $\mathrm{T} 2$ with $\mathrm{T} 0$ ).

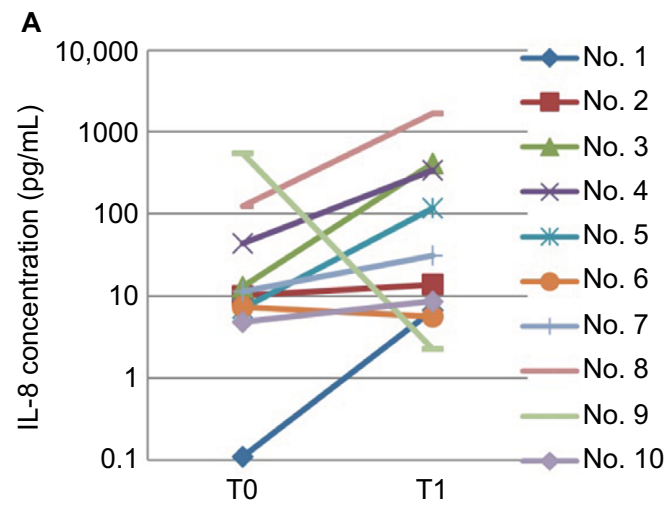

The increase in the serum IL- 12 level induced by O3-AHT did not reach statistical significance

We then analyzed the effect of O3-AHT on serum IL-12 level. As shown in Figure 2 and Table 2, after five courses of O3-AHT (T1), the average serum IL-12 level was increased from 1.66 to $2.06 \mathrm{pg} / \mathrm{mL}(Z=0.135, P=0.893)$, but the difference was not statistically significant. After completing the treatment (T2), the level was decreased to $1.50 \mathrm{pg} / \mathrm{mL}$ at T2 ( $Z=0.405, P=0.686$, compared to T0), which was lower than that at T0. However, the difference was not statistically significant either.

\section{O3-AHT did not change the serum MCP-I level in gouty patients}

We further examined the serum level of MCP-1 during and after O3-AHT. Our results show that, after the 5th course of O3-AHT (T1), the MCP-1 was only slightly decreased compared to the level prior to O3-AHT $(92.87 \mathrm{pg} / \mathrm{mL}$ at T1 relative to $95.89 \mathrm{pg} / \mathrm{mL}$ at $\mathrm{T} 0, Z=0.459, P=0.646$ ). The MCP-1 level after completing the treatment (T2) was $95.02 \mathrm{pg} / \mathrm{mL}$, which was essentially not different from the level before O3-AHT ( $Z=0.770, P=0.441$, compared to T0) (Table 2 and Figure 2).

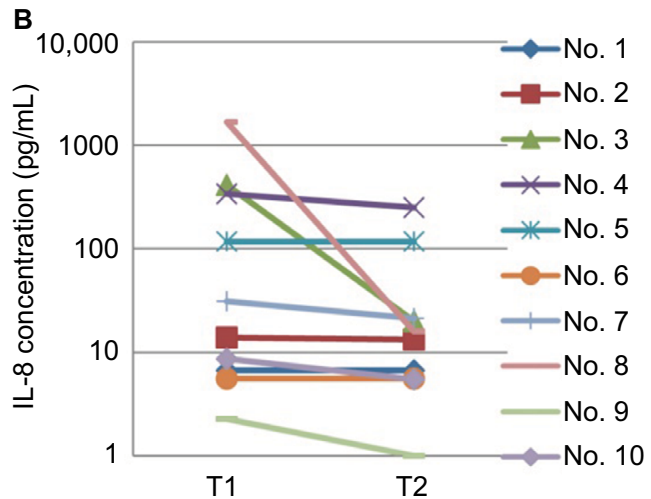

Figure 2 Changes of serum IL-8 levels in gouty patients following O3-AHT.

Notes: (A) Comparison of serum IL-8 levels in patients after the 5th course of O3-AHT (TI) with those in patients prior to treatment (T0). (B) Comparison of serum IL-8 levels in patients after completing O3-AHT (T2) with those in patients after the 5th course of O3-AHT (TI).

Abbreviation: O3-AHT, ozonated autohemotherapy.

Table 2 Changes in serum cytokines in gouty patients treated with O3-AHT

\begin{tabular}{|c|c|c|c|c|c|c|}
\hline $\begin{array}{l}\text { Time } \\
\text { point }\end{array}$ & IL-8 & $\begin{array}{l}\text { Wilcoxon test } \\
\text { (compared to T0) }\end{array}$ & MCP-I & $\begin{array}{l}\text { Wilcoxon test } \\
\text { (compared to T0) }\end{array}$ & IL-I 2 & $\begin{array}{l}\text { Wilcoxon test } \\
\text { (compared to T0) }\end{array}$ \\
\hline$\overline{\mathrm{TO}}$ & $77.95 \pm 171.95$ & - & $95.89 \pm 52.00$ & - & $1.66 \pm 2.7 \mathrm{I}$ & - \\
\hline TI & $262.52 \pm 521.06$ & $Z=2.487, P=0.013$ & $92.87 \pm 41.17$ & $Z=0.459, P=0.646$ & $2.06 \pm 4.16$ & $Z=0.135, P=0.893$ \\
\hline $\mathrm{T} 2$ & $45.83 \pm 79.88$ & $Z=1.376, P=0.169$ & $95.02 \pm 43.86$ & $Z=0.770, P=0.44 \mathrm{I}$ & $1.50 \pm 3.32$ & $Z=0.405, P=0.686$ \\
\hline
\end{tabular}

Abbreviation: O3-AHT, ozonated autohemotherapy. 


\section{Discussion}

The development of gout is a multistep inflammatory process, which is triggered by interactions between MSU crystals and local microenvironment. Disorder of inflammatory cytokines plays a crucial role in gouty inflammation. ${ }^{11,16-18}$ Antiinflammatory drug approaches have been used to treat gouty patients. ${ }^{19,20}$ In this study, to investigate the effect of O3-AHT on gouty inflammation, cytometric bead array was used to measure a group of cytokines related to gouty inflammation. Our results show that the serum IL-8 level was significantly increased after the 5th course of O3-AHT (T1). This is consistent with previous findings that ozonation enhances the release of IL-8 in cultured human umbilical vein endothelial cells. ${ }^{21}$

The critical role of IL-8 in gout has been demonstrated in previous studies. For example, Kienhorst et $\mathrm{al}^{11}$ recently reported that the serum levels of IL-8/CXCL8 are increased during both the acute and intercritical phases of gout. Terkeltaub et $\mathrm{al}^{22}$ have demonstrated that IL-8 and its related cytokines, CXCL1, could bind to CXCR2 to promote chemotaxis of neutrophils during gouty inflammation in mouse model. In addition, Nishimura et al ${ }^{23}$ showed that neutrophil influx phase was suppressed when using an antibody of IL-8 to treat MSUinduced arthritis in a rabbit model. Furthermore, genotyping research revealed that polymorphism of IL-8 251T/A and IL12B 1188A/C might be associated with susceptibility of gout development. ${ }^{24}$ The sensitive response of serum IL-8 level to O3-AHT certainly suggests that O3-AHT is able to modulate the inflammation status by modulating the expression of this key gouty-associated cytokine. One of the IL-8 functions is to induce phagocytosis once it has arrived at the inflammation site. ${ }^{25}$ The increased concentration of IL- 8 in the gouty inflammation site may promote apoptotic cell clearance, thereby leading to the reduction in inflammation burden. Therefore, the increase in IL-8 level might be one of the mechanisms by which O3-AHT reduces the gouty inflammation, which may subsequently contribute to the pain relief induced by O3-AHT.

Many questions need to be answered regarding the modulation of IL-8 level by O3-AHT. For example, does O3-AHT directly modulate IL-8 protein expression, or does it only enhance the mobilization of IL-8 to the circulation system? Does O3-AHT increase the level of IL-8 at the local inflammation site? Does the increased IL-8 contribute to the relief of local inflammation and pain? Intensive studies are certainly needed to answer these questions in the future.

Similar to the response of serum IL-8 level to O3-AHT, the serum IL-12 level was also increased after five courses of O3-AHT (T1). The difference only reached marginal statistical significance, which might be due to the small sample size. This is also consistent with previous findings that O3-AHT at the concentration of $30-55 \mu \mathrm{g} / \mathrm{mL}$ could promote IL-12 production by increasing the levels of reactive oxygen species (ROS) and lipid oxidation products (LOPs), which are two messengers produced during the interaction of ozone and blood. ${ }^{21}$ Similarly, the role of IL-12 in mediating the therapeutic effect of O3-AHT also needs to be further investigated in the future.

In addition, we observed that, following completion of O3-AHT, both IL-8 and IL-12 decreased to levels lower than before O3-AHT. One of the explanations is that inflammation is dramatically reduced by multiple courses of O3-AHT, which leads to the feedback downregulation of IL- 8 and IL-12 expressions after the full courses of O3-AHT.

MCP-1 was also reported to be closely associated with the inflammation process of gout. Grainger et $\mathrm{al}^{26}$ demonstrated that hyperuricemia causes an elevated level of MCP-1. Scanu et $\mathrm{al}^{27}$ revealed that MSU crystal induces fibroblast-like synoviocytes to release MCP-1. Consistent with previous findings, we show that the serum MCP-1 level was increased compared with hyperuricemia patients and healthy controls, although the differences did not reach statistical significance. However, the MCP-1 level in gouty patients was not significantly affected by O3-AHT, suggesting that MCP-1 may not play a key role in the therapeutic mechanism of O3-AHT.

Unexpectedly, we found that the concentrations of the rest of the nine cytokines that we examined were not detectable in all the three groups and under all treatment conditions. This is highly likely due to the insufficient sensitivity of measurement approaches for these specific cytokines. Future studies are certainly needed to further examine the levels of these cytokines in gouty patients and to evaluate the effect of O3-AHT on their expressions, by exploiting more sensitive measurement approaches.

There are several limitations in our study. In this study, we observed that the increase in serum IL-12 levels after the 5th course of O3-AHT did not reach statistical significance. This is highly likely due to the small sample size. In addition, although the effect of O3-AHT on the serum IL-8 levels reached statistical significance, the statistical power of the study was low due to the small sample size. Future studies in larger patient cohorts are certainly needed to further evaluate the effect of O3-AHT on IL-12 expression. In addition, with this sample size, a double-blind randomized controlled trial could not be performed. Moreover, nine cytokines were not detectable using the current approach. It is possible that the expression of these cytokines might also be modulated by O3-AHT. These cytokines warrant further investigations by exploiting more sensitive measurement approaches. 
Overall, our results indicate that, among all the cytokines that we examined, IL- 8 and IL-12 stand out as the most sensitive response to $\mathrm{O} 3-\mathrm{AHT}$. On one hand, these findings suggest that O3-AHT might alleviate gouty inflammation and relieve pain by regulating the expression of crucial inflammation cytokines IL-8 and IL-12. Our study paved a way to further determine the role of inflammation cytokines in mediating the therapeutic effect of O3-AHT in gouty patients. On the other hand, the sensitive response of IL- 8 and IL-12 to O3-AHT suggests that IL- 8 and IL-12 may have the potential to be developed as molecular markers for evaluating the effectiveness of O3-AHT.

In the future, investigating the effect of O3-AHT on serum levels of IL-8 and IL-12 in a larger group of patients and determining the correlation of the serum levels of IL-8 and IL-12 with the therapeutic efficacy of O3-AHT would help to further define whether IL-8 and IL-12 play a role in O3-AHT. From the therapeutic perspective, whether combined O3-AHT and anti-inflammatory treatment have synergistic therapeutic effect could also be explored.

\section{Disclosure}

The authors report no conflicts of interest in this work.

\section{References}

1. Busso N, So A. Mechanisms of inflammation in gout. Arthritis Res Ther. 2010;12(2):206.

2. Tausche AK, Jansen TL, Schröder HE, Bornstein SR, Aringer M, MüllerLadner U. Gout - current diagnosis and treatment. Dtsch Arztebl Int. 2009;106(34-35):549-555.

3. McClory J, Said N. Gout in women. Med Health R I. 2009;92(11):363-364.

4. Choi HK. A prescription for lifestyle change in patients with hyperuricemia and gout. Curr Opin Rheumatol. 2010;22(2):165-172.

5. Choi HK, Zhu Y, Mount DB. Genetics of gout. Curr Opin Rheumatol. 2010;22(2):144-151.

6. Roddy E, Choi HK. Epidemiology of gout. Rheum Dis Clin North Am. 2014;40(2):155-175.

7. Burns CM, Wortmann RL. Gout therapeutics: new drugs for an old disease. Lancet. 2011;377(9760):165-177.

8. Zaky S, Kamel SE, Hassan MS, et al. Preliminary results of ozone therapy as a possible treatment for patients with chronic hepatitis C. $J$ Altern Complement Med. 2011;17(3):259-263.

9. Ripamonti CI, Cislaghi E, Mariani L, Maniezzo M. Efficacy and safety of medical ozone $(\mathrm{O}(3))$ delivered in oil suspension applications for the treatment of osteonecrosis of the jaw in patients with bone metastases treated with bisphosphonates: preliminary results of a phase I-II study. Oral Oncol. 2011;47(3):185-190.

10. Li LY, Ni JX. Efficacy and safety of ozonated autohemotherapy in patients with hyperuricemia and gout: a phase I pilot study. Exp Ther Med. 2014;8(5):1423-1427.
11. Kienhorst LB, van Lochem E, Kievit W, et al. Gout is a chronic inflammatory disease in which high levels of interleukin-8 (CXCL8), myeloidrelated protein $8 /$ myeloid-related protein 14 complex, and an altered proteome are associated with diabetes mellitus and cardiovascular disease. Arthritis Rheumatol. 2015;67(12):3303-3313.

12. Shaw OM, Steiger S, Liu X, Hamilton JA, Harper JL. Brief report: granulocyte-macrophage colony-stimulating factor drives monosodium urate monohydrate crystal-induced inflammatory macrophage differentiation and NLRP3 inflammasome up-regulation in an in vivo mouse model. Arthritis Rheumatol. 2014;66(9):2423-2428.

13. Tausche AK, Richter K, Grassler A, et al. Severe gouty arthritis refractory to anti-inflammatory drugs: treatment with anti-tumour necrosis factor alpha as a new therapeutic option. Ann Rheum Dis. 2004;63(10):1351-1352.

14. Fiehn C, Zeier M. Successful treatment of chronic tophaceous gout with infliximab (Remicade). Rheumatol Int. 2006;26(3):274-276.

15. Zhang W, Doherty M, Pascual E, et al. EULAR evidence based recommendations for gout. Part I: diagnosis. Report of a task force of the Standing Committee for International Clinical Studies Including Therapeutics (ESCISIT). Ann Rheum Dis. 2006;65(10):1301-1311.

16. Mitroulis I, Kambas K, Ritis K. Neutrophils, IL-1beta, and gout: is there a link? Semin Immunopathol. 2013;35(4):501-512.

17. Torres R, Macdonald L, Croll SD, et al. Hyperalgesia, synovitis and multiple biomarkers of inflammation are suppressed by interleukin 1 inhibition in a novel animal model of gouty arthritis. Ann Rheum Dis. 2009;68(10):1602-1608.

18. Cavalcanti NG, Marques CD, Lins ELTU, et al. Cytokine profile in gout: inflammation driven by IL-6 and IL-18? Immunol Invest. 2016;45(5):383-395.

19. van Durme CM, Wechalekar MD, Landewe RB. Nonsteroidal antiinflammatory drugs for treatment of acute gout. JAMA. 2015;313(22): 2276-2277.

20. Richette P, Frazier A, Bardin T. Impact of anti-inflammatory therapies, xanthine oxidase inhibitors and other urate-lowering therapies on cardiovascular diseases in gout. Curr Opin Rheumatol. 2015;27(2):170-174.

21. Bocci V, Valacchi G, Corradeschi F, et al. Studies on the biological effects of ozone: 7. Generation of reactive oxygen species (ROS) after exposure of human blood to ozone. J Biol Regul Homeost Agents. 1998;12(3):67-75.

22. Terkeltaub R, Baird S, Sears P, Santiago R, Boisvert W. The murine homolog of the interleukin- 8 receptor CXCR-2 is essential for the occurrence of neutrophilic inflammation in the air pouch model of acute urate crystal-induced gouty synovitis. Arthritis Rheum. 1998;41(5):900-909.

23. Nishimura A, Akahoshi T, Takahashi M, et al. Attenuation of monosodium urate crystal-induced arthritis in rabbits by a neutralizing antibody against interleukin-8. J Leukoc Biol. 1997;62(4):444-449.

24. Liu S, Yin C, Chu N, Han L, Li C. IL-8 -251T/A and IL-12B 1188A/C polymorphisms are associated with gout in a Chinese male population. Scand J Rheumatol. 2013;42(2):150-158.

25. Beste MT, Lomakina EB, Hammer DA, Waugh RE. Immobilized IL-8 triggers phagocytosis and dynamic changes in membrane microtopology in human neutrophils. Ann Biomed Eng. 2015;43(9):2207-2219.

26. Grainger R, McLaughlin RJ, Harrison AA, Harper JL. Hyperuricaemia elevates circulating CCL2 levels and primes monocyte trafficking in subjects with inter-critical gout. Rheumatology (Oxford). 2013;52(6):1018-1021.

27. Scanu A, Oliviero F, Gruaz L, et al. High-density lipoproteins downregulate CCL2 production in human fibroblast-like synoviocytes stimulated by urate crystals. Arthritis Res Ther. 2010;12(1):R23. 
Open Access Rheumatology: Research and Reviews is an international, peerreviewed, open access journal publishing original research, reports, editorials, reviews and commentaries on all aspects of clinical and experimental rheumatology in the clinic and laboratory including the following topics: Pathology, pathophysiology of rheumatological diseases; Investigation, treatment and management of rheumatological diseases; Clinical trials and novel pharmacological approaches for the treatment of rheumatological disorders. The manuscript management system is completely online and includes a very quick and fair peer-review system, which is all easy to use. Visit http://www.dovepress.com/ testimonials.php to read real quotes from published authors. 